Sādhanā Vol. 40, Part 7, October 2015, pp. 2163-2176. (C) Indian Academy of Sciences

\title{
Spatial resolution enhancement residual coding using hybrid wavelets and directional filter banks
}

\author{
ANKIT ASHOKRAO BHURANE*, PRATEEK CHAPLOT, \\ DUSHYANTH NUTULAPATI and VIKRAM M GADRE
}
Department of Electrical Engineering, Indian Institute of Technology Bombay, Powai, Mumbai 400 076, India
e-mail: ankit.bhurane@gmail.com; pratchap@gmail.com;
dushyanth.iitb@gmail.com; vmgadre@ee.iitb.ac.in

MS received 28 February 2014; revised 07 November 2014; accepted 12 November 2014

\begin{abstract}
Traditional video coding uses classical predictive coding techniques, where a signal is initially approximated by taking advantage of the various redundancies present. Most of the video coding standards, including the latest HEVC, use the well-accepted procedure of applying transform coding on self-contained (intra) and inter-predicted frame residuals. Nevertheless, it has been shown in the literature that, a normal video frames possess distinct characteristics compared to a residual frame. In this paper, we have made use of hybrid wavelet transforms and directional filter banks (HWD) to encode resolution enhancement residuals in the context of scalable video coding. The results are presented for the use of HWD in the framework of the Dirac video codec. The experiments are carried out on a variety of test frames. Our experiments on residue coding using HWD show better performance compared to the conventional DWT, when tested on the same platform of the well-known SPIHT algorithm.
\end{abstract}

Keywords. Residual; Dirac video codec; HWD; SPIHT.

\section{Introduction}

Video coding research has progressed tremendously over the past couple of decades, thanks to the increasing demands of video communication that motivates researchers to develop cuttingedge algorithms. All the video coding standards, to date, make use of various predictive coding techniques to remove redundancies. The procedure broadly follows the same queue of predictive coding, transform on residuals, quantization and entropy coding to minimize spatiotemporal, intra-frame, visual, and statistical redundancies, respectively. Intra and inter prediction

*For correspondence 
algorithms themselves put a limit on encoding efficiency to a certain extent, after which approximation becomes difficult (Ya-quin Zhang et al 2001). State-of-the art quantization uses uniform quantization with scaling factors defined as per the standards (Sullivan et al 2012) and entropy coding uses context-adaptive arithmetic coding offering efficiency close to the entropy limit. Residuals are the product of various prediction techniques, depending on which they are broadly classified as intra-prediction (IP), motion compensation (MC), and spatial resolution enhancement (RE) or inter-scale residuals. The IP and MC residuals are part of a typical video codec, while RE residuals are specific to the spatially scalable extension of a video codec. Our discussion is focused on the encoding of the RE residual. However, the concept can also be extended for MC and IP residuals. Residuals have their histograms concentrated at the center showing little variation in the pixel intensities; normal video frames may have their histograms spread over many intensities. The nature of residuals coupled with the intuition of a researcher in the field suggests the use of transforms with a large set of directions rather than the conventional discrete cosine or wavelet transforms. This can help in approximating the content of residuals with fewer coefficients and gives better coding efficiency.

The outline of this paper is as follows. Section 2 briefly outlines the related work and modelling of residuals. Section 3 builds the concept of scalability in a video, in general. Section 4 gives an overview of the Dirac video codec, and our attempt to extend it for intra-only spatial scalability. Section 5 describes the HWD-H transform in brief. Experimental results for residual coding are given in section 6. Finally, conclusions are drawn in section 7.

\section{Related work}

Kamisli \& Lim made use of 1D directional, adaptive DCTs (Kamisli \& Lim 2009b) and 1D directional DWT (Kamisli \& Lim 2009a) to encode IP and MC residuals. Their results claim better performance when 1D directional DCTs are incorporated in the H.264 framework. However, the performance for RE residuals was not evaluated. The work was further extended by Cohen et al in the year 2010 by proposing Direction-Adaptive Residual Transform (DART) (Cohen et al 2010), where 1D DCT was again applied over the DC coefficients generated by the 1D directional DCTs. Although the results shown are better when compared to the traditional 2D DCT, these approaches are adaptive and need several checks to get the best directional basis. Temizel \& Vlachos (2005) proposed a resolution enhancement technique using an average of a set of multiple low-resolution images obtained by multiple shifts. Chang et al (1995) proposed a wavelet-based iterative approach for enhancing the image resolution. Recently, Kang et al (2013) proposed a two-layer transform for coding the residuals obtained from the HEVC architecture. The first stage makes use of a pre-trained dictionary to get a sparse representation of the residuals. The residuals obtained after the sparse representation are then coded using the DCT. This approach is also adaptive and complex. Jianping et al (2005) proposed a non-subsampled contourlet transform, which is a non-subsampled pyramid formation followed by directional filter banks, for contour enhancement. Although the proposed approach is shift-invariant with an application for edge enhancement, the transform is redundant due to the absence of downsamplers. We have made use of a hybrid wavelet transform and directional filter banks (HWD) for encoding the RE residuals. The HWD transforms are non-redundant, non-adaptive, have better nonlinear approximation and flexibility. The details of HWD are given in section 6 . The advantages of HWD over the contourlet transform are clearly mentioned and discussed in the work by Eslami \& Radha $(2007,2006)$. 


\section{Characterization of residuals}

Residual frames possess different statistical properties in comparison with normal video frames. The characteristics of residuals are extensively studied in the literature. To list a few studies, Chen \& Pang (1993) proposed a statistical model for motion-compensated residuals. Secondorder statistics using for MC residuals were analysed by Niehsen \& Brunig (1999).

Kamisli et al in the year 2009 investigated the use of a generalized, covariance model to analyze the characteristics of IP, MC and RE residuals (Kamisli 2010; Rao et al 2007). The estimation used is unbiased, which considers only the total number of overlapping points during estimation. The generalized covariance model is given by the following equation:

$$
C(\theta, I, J)=\rho_{1}^{|I \cos (\theta)+J \sin (\theta)|} \rho_{2}^{|-I \sin (\theta)+J \cos (\theta)|},
$$

where $(|I|,|J|)$ are the lags for estimation, $\theta$ is the axis rotation angle and $\left(\rho_{1}, \rho_{2}\right)$ are the positive correlation coefficient along rows and columns of an image, respectively. For a signal $x(n)$ under consideration, $\rho$ can be calculated using the following equation

$$
\rho(k)=\frac{\frac{1}{N-|k|} \sum_{n=0}^{N-|k|-1} x(n) x(n+|k|)}{\sigma_{x}^{2}},
$$

where, $k$ is the separation between two samples, called as lag and $\sigma_{x}^{2}$ is the zero-mean variance of the samples under consideration. Algorithm 1 shows the details of the estimation process.

Given: $7 \times 7$ block

Calculate autocorrelation $A$

for $0 \leq \theta<\pi$ do

for $0<\rho_{1}<1$ do

for $0<\rho_{2}<1$ do

$$
\text { for }-3 \leq I \leq 3 \text { do }
$$

$$
\text { for }-3 \leq J \leq 3 \text { do }
$$

$$
C(\theta, I, J)=\rho_{1}^{|I \cos (\theta)+J \sin (\theta)|} \cdot \rho_{2}^{|-I \sin (\theta)+J \cos (\theta)|}
$$

\section{end}

$\mathrm{E}=\operatorname{mse}(A, C)$

$$
\text { end }
$$

end

$$
\text { end }
$$

end

$\arg \min _{\left(\rho_{1}, \rho_{2}, \theta\right)} E$

Output: $\left(\rho_{1}, \rho_{2}, \theta\right)$

Algorithm 1. Pseudo-code for modelling 2D signals. 
The explanation of the algorithm is as follows. We generate a $7 \times 7$ matrix $C$ for every possible value of the parameters $\left(\rho_{1}, \rho_{2}, \theta\right)$ in their respective ranges. For each block, we then choose one of these which captures the block autocorrelation in the best manner. The criterion for best fit is the mean square error between the estimated and actual autocorrelation coefficients. Having obtained the best fit, the $\left(\rho_{1}, \rho_{2}\right)$ pair is sorted so that the left element is greater than or equal to the right element. The scatter plots are then plotted against these two variables. To observe the characteristics of residuals, consider the example shown in figure 1. The scatter plots represent the best fit $\left(\rho_{1}, \rho_{2}\right)$ for a frame and its RE residual. The plots clearly show different characteristics for the frame and corresponding residual, where the residual has the density of points oriented towards bottom right corner specifying directional content. For better interpretation, the mean of density of scatter plots is marked. The closer the mean is to the reference point $(1,0)$, more is the directional content in the signal.

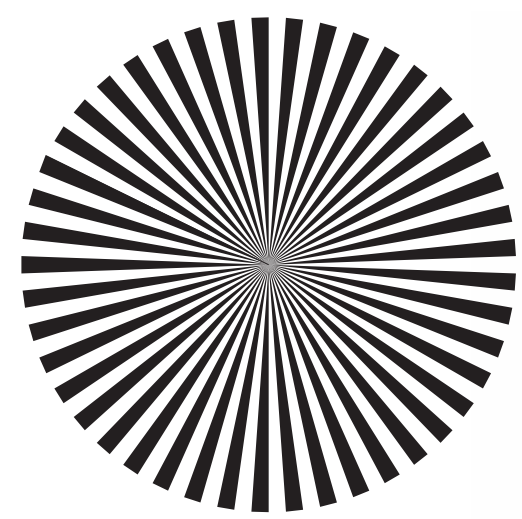

(a) Spokes image [online]

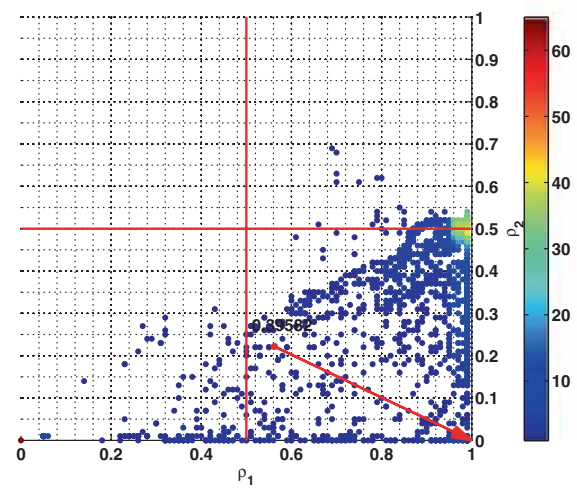

(c) Scatter plot for (a)

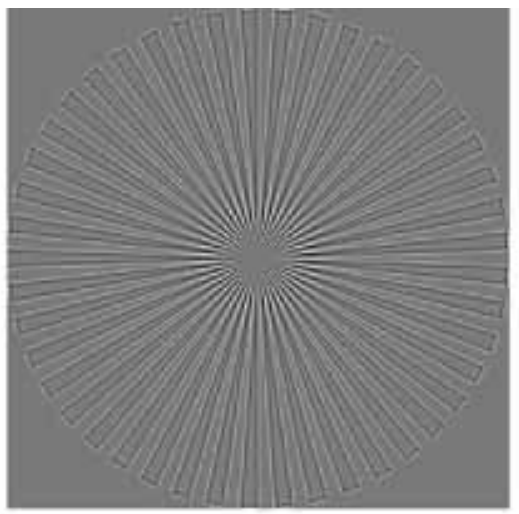

(b) RE residual of (a)

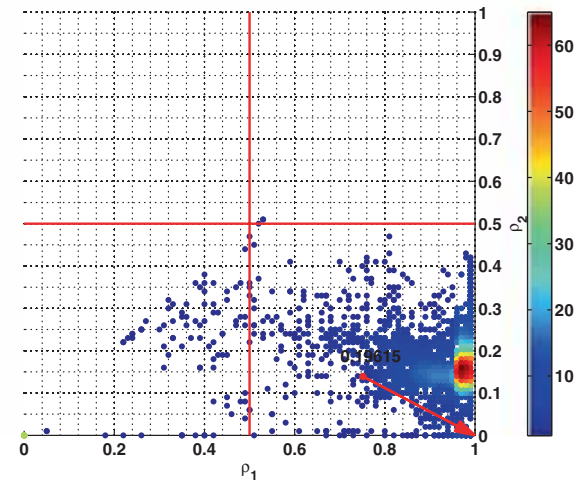

(d) Scatter plot for (b)

Figure 1. Characterization of test signal. Each point in the plot is constructed from the two coefficients $\left(\rho_{1}, \rho_{2}\right)$ that best fits the estimation model. The arrow annotation shows the Euclidean distance between the reference point $(1,0)$ and the mean of all the scatter points. The colour bar indicates the density of a set of points in a region. 


\section{Scalability in a video}

Scalability is a feature of a bit stream which provides the facility to cater to the needs of various heterogeneous devices. There can be different variants of scalability. The most commonly used are: spatial, temporal, quality, and a combination of these. The original video can be represented in a layered form of scalability as follows. A layered bit stream consists of an essential base layer, which represents the lowest resolution supported by the bit stream. The higher layers are initially predicted from preceding lower layers using inter-layer prediction functions. Further, the predicted information is combined with the incremental information to represent higher resolutions. The successive layers after the base layer are called as enhancement layers. Therefore, the base layer is self-contained, while enhancement layers are dependent on all the preceding layers including the base layer. The number of layers for different types of scalabilities may not be equal. Usually, the ratio between resolutions obtained by two successive layers are fixed to be powers of two. This is known as dyadic scalability. For extended scalability, the scaling ratios need not be in powers of two (Segall \& Sullivan 2007). Scalability in the DCT-based video codecs is usually incorporated as an extension to it, whereas wavelet-based video codecs take the advantage of their inherent multiresolution nature to provide the desired facility.

The problem of scalable video compression is to minimize the gap between the performance curves (PSNR or SSIM) for single layer encoding and its equivalent scalable bit stream for scalability to be a meaningful choice. The performance of the scalable bit stream must always be better than its equivalent simulcast bit stream. This is achieved at the cost of acceptable increase in the complexity. The next section describes a simple extension for spatial scalability to an open-source video codec.

\section{Dirac video codec and its intra-only, spatially scalable extension}

Increasing patents and royalty costs have motivated researchers to develop open-source video codecs. Efforts are made using both DCT and wavelet-based techniques, a few include Theora (Theora video codec [online]. http://www.theora.org/. Accessed: 2013-07-29), ongoing WebM (Theora video codec [online]. http://www.webmproject.org. Accessed: 2013-10-18) and Dirac (Dirac video codec [online]. http://diracvideo.org/. Accessed: 2013-07-29). Dirac is one of the several attempts to incorporate the wavelet transform as the crux of a video codec. Dirac was developed by BBC and aimed at a royalty-free, open technology (Dirac video codec [online]. http://diracvideo.org/. Accessed: 2013-07-29). The intra-only version of Dirac is also recently standardized as the VC-2 standard. In this version, the frames are coded without using any reference frames. The performance of the Dirac video codec was also reported to be promising in comparison with the state-of-the-art DCT-based H.264 (Kalra et al 2011). Dirac uses hierarchical overlap block motion compensation (OBMC), DWT, integer approximated dead-zone quantization and context adaptive arithmetic coding (Dirac specification version 2.2.3 [online]. http://diracvideo.org/download/specification/dirac-spec-latest.pdf. Accessed: 2013-07-29). The block diagram of Dirac video codec is shown in figure 2.

We have extended the Dirac video codec to facilitate an intra-only spatial scalability. For downsampling, the filter defined in MPEG-4, having a point spread function given by $[2,0,-4,-3,5,19,26,19,5,-3,-4,0,2] / 64$ is used. For upsampling, AVC interpolation filters with coefficients $[1,-5,20,20,-5,1] / 32$ and $[16,16] / 32$ are used for luma and croma pixels, respectively. The resampling filters are chosen as per the recommendations of Joint Scalable 


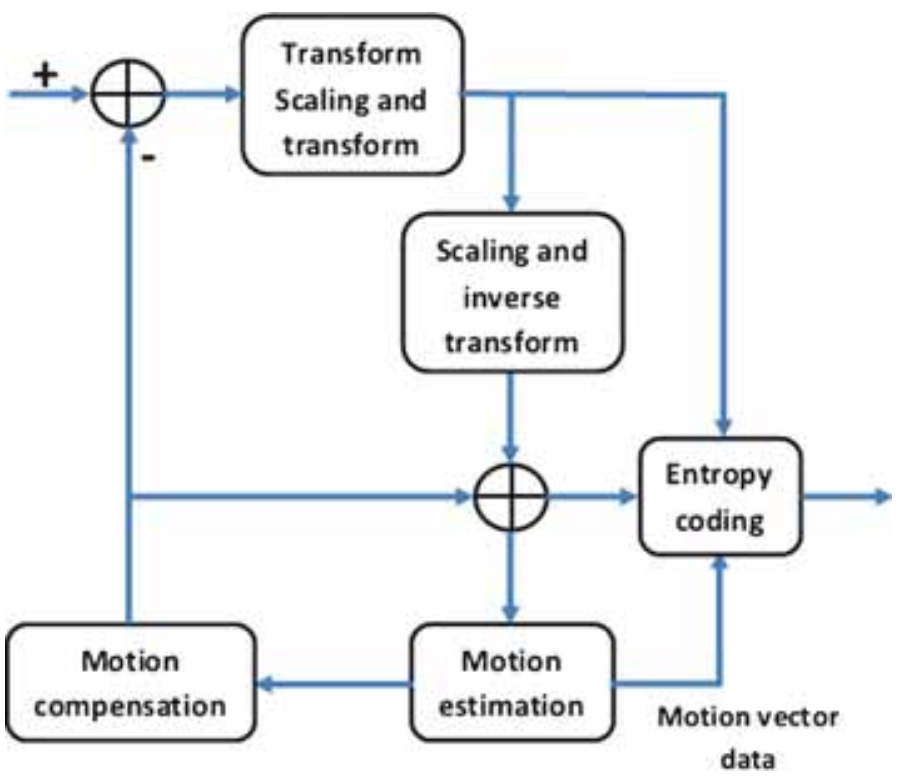

Figure 2. Dirac video codec architecture.

Video Model (JSVM), a scalable extension of H.264 (Jsvm reference software [online]. http://vwww.hhi.fraunhofer.de/en/departments/image-processing/image-vide\%o-coding/svcextension-of-h264avc/jsvm-reference-software/. Accessed: 2013-10-18; Schwarz et al 2007. The residuals obtained after rescaling of the locally decoded frame by spatial scalable decoding, are RE residuals, which are then encoded using hybrid wavelet transform and directional filter banks (HWD) as shown in figure 3. In this paper, we restrict our discussion to the encoding of $\mathrm{RE}$ residuals obtained in such architectures.

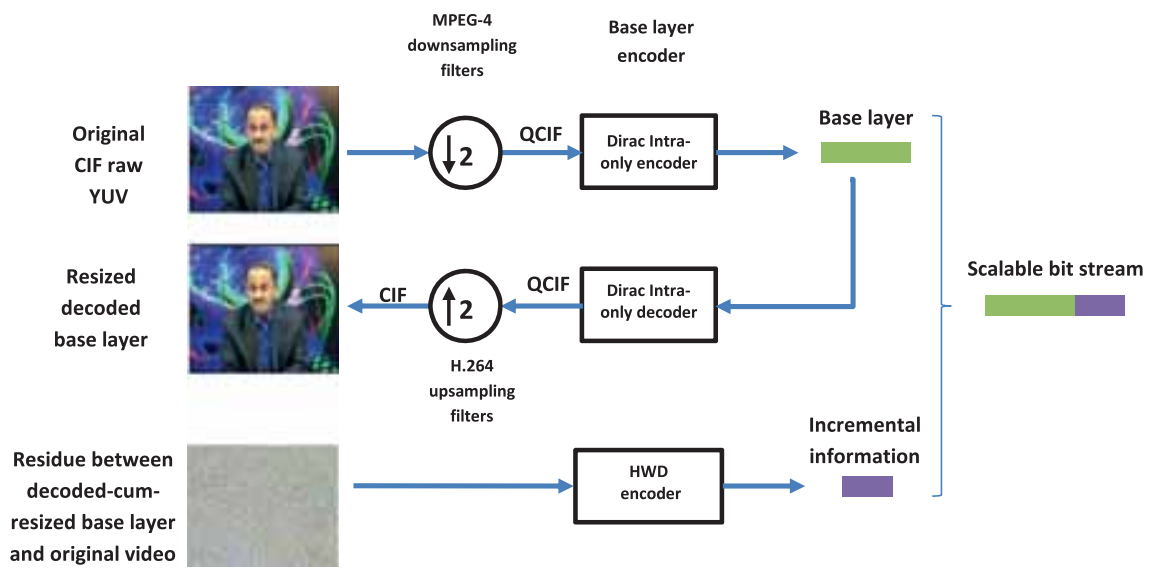

Figure 3. Integrating spatial scalability with Dirac video codec. 


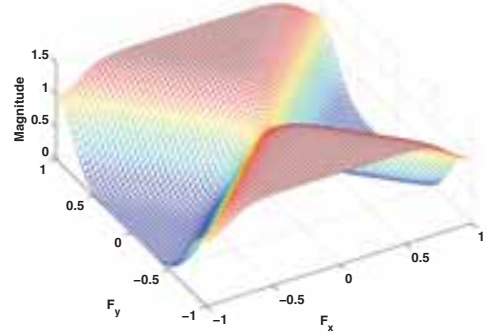

(a)

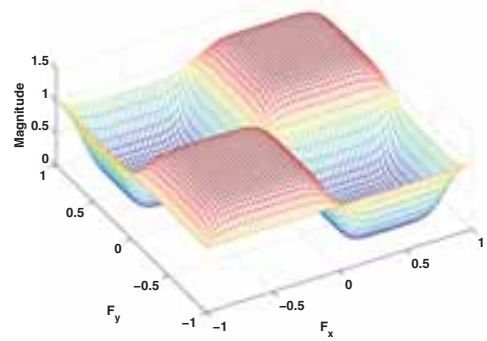

(c)

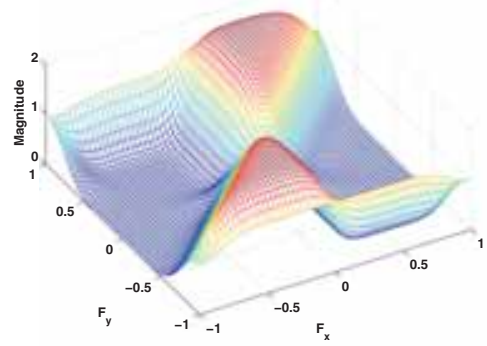

(e)

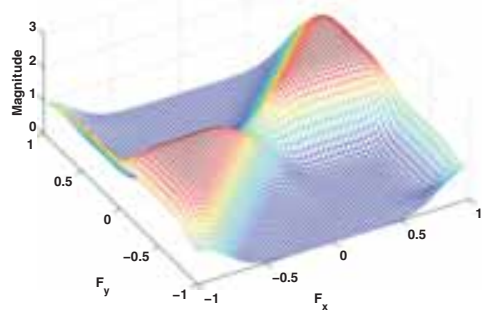

(g)

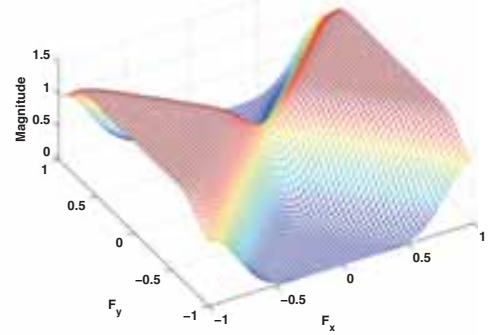

(b)

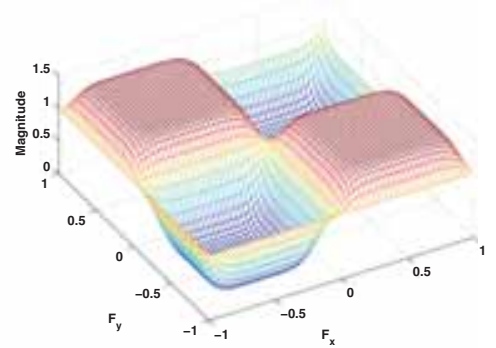

(d)

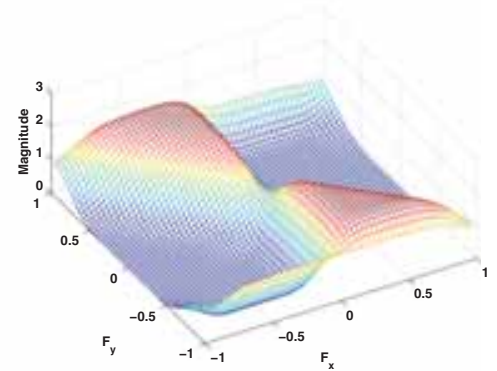

(f)

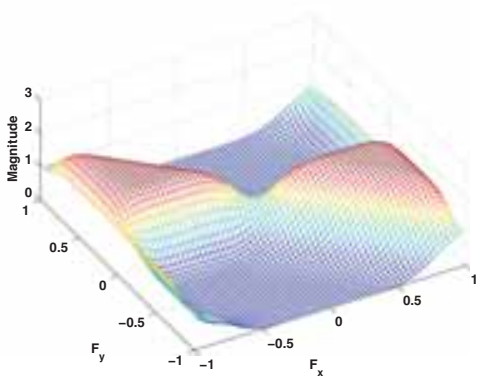

(h)

Figure 4. Frequency response of (a) and (b) fan filters, (c) and (d) quadrant filters and (e)-(h) wedge filters for HWD-H obtained from combination of fan and quadrant filters. 


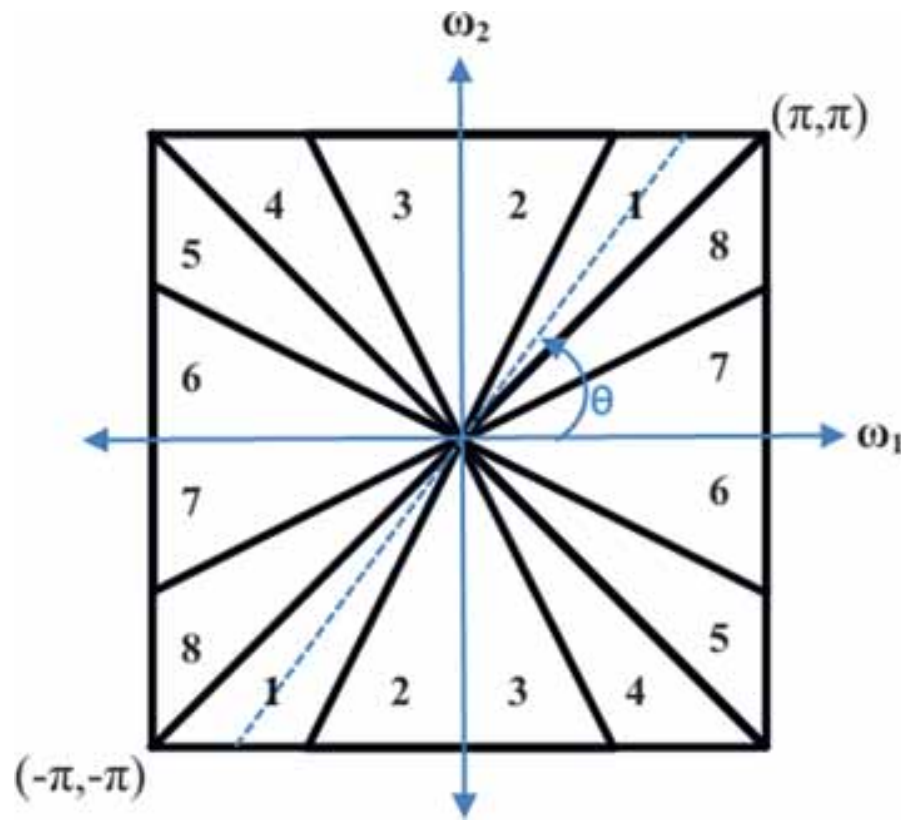

Figure 5. Orientation and division of frequency space for three levels. $(1,2,3,4)$ only represents frequency partitions for horizontal directional filter bank and $(5,6,7,8)$ only represents frequency partitions for vertical filter bank.

\section{The hybrid wavelet transform and directional filter banks (HWD)}

The RE residuals obtained from spatial scalable decoding possess contours (see figure 1b). These patterns suggest the use of directional transforms which could capture the contour details in fewer coefficients. Many of the available directional transforms like contourlets, dual-tree complex wavelet transforms (Selesnick et al 2005), are redundant or adaptive or have a limited set of directions.

HWD is a class of non-redundant, directional, hybrid wavelet transforms defined by Eslami et al. In contrast with the specifically designed 1D directional DCT and wavelet transforms proposed by Kamisli et al in the year 2007, HWD operates on the complete residual frame instead of block-wise processing. HWD is a two-tier transform, where initially a conventional DWT is applied, followed by the directional filters on the detail subbands. In Eslami \& Radha (2006), three types of directional transforms were introduced. In type-1, subbands with vertical (horizontal) directional information are subjected to further decomposition using vertical (horizontal) directional filter banks. In type-2, subbands with vertical (horizontal) directional information are subjected to further decomposition using horizontal (vertical) directional filter banks. In type-1 and type-2, full directional filter banks are applied to only (diagonal) HH subbands whereas in type-3, full directional filter banks are applied to all the subbands, irrespective of their directions. In this paper, we have made use of type-2 HWD namely, HWD half-tree (HWD-H). The motivation for using type-2 HWD is to reduce the effect of frequency scrambling. A detailed discussion on the effect of frequency scrambling is reported in Eslami \& Radha (2007). The kernel low-pass impulse response used to obtain the set of directional filters is 
$T=[-0.0024,0.0239,-0.1196,0.5981,0.5981,-0.1196,0.0239,-0.0024]$. The filter pairs obtained from $T$ have a frequency response as shown in figure 4 . The directional filters have wedge-shaped structures and are obtained by combining the frequency responses of corresponding fan and quadrant filters. Frequency responses in figures $4 \mathrm{e}$ and $\mathrm{f}$ are obtained by multiplying frequency responses in figures $4 \mathrm{a}, \mathrm{c}$, and $\mathrm{d}$, respectively. Also similar operations are performed for frequency responses in figures $4 \mathrm{~g}$ and $\mathrm{h}$.

The orientation of the frequency support for $L$ level decomposition into horizontal and vertical directional filter banks is defined as Li Ma et al (2010)

$$
\theta(i)=\left\{\begin{array}{cl}
\left(\frac{1}{4}-\frac{1}{2^{L+1}}+\frac{i}{2^{L}}\right) \pi & ; \text { for horizontal } \\
\left(\frac{1}{4}-\frac{1}{2^{L+1}}+\frac{i}{2^{L}}\right) \pi-\frac{\pi}{2} & ; \text { for vertical }
\end{array}\right.
$$

where $i$ takes the values $1 \leq i \leq 2^{L-1}$. Figure 5 shows the frequency orientation for 3-levels of directional decomposition.

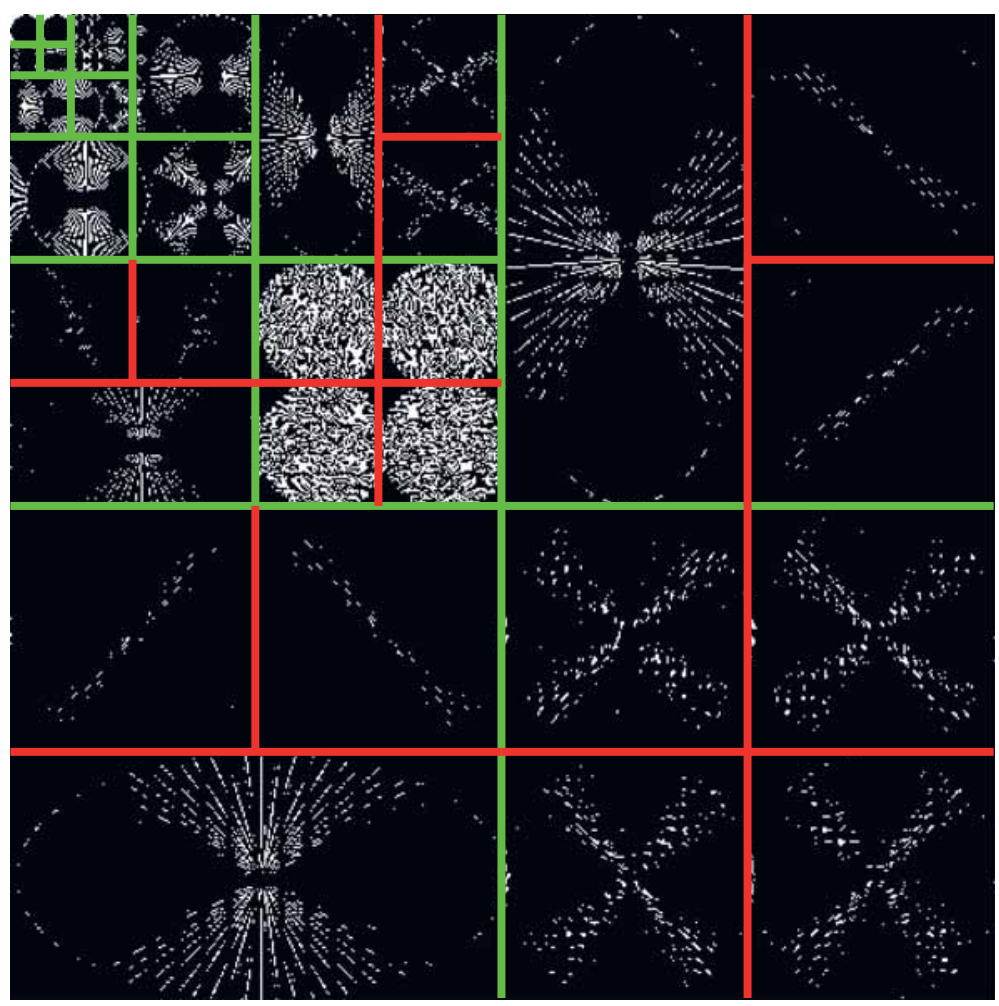

Figure 6. An example of applying the HWD-H transform on Spokes image. HWD on Spokes image indicates DWT partitioning (green) and HWD partitioning (red) for decomposition vector $[2,2,0,0,0]$. The image is thresholded for better visualization. 


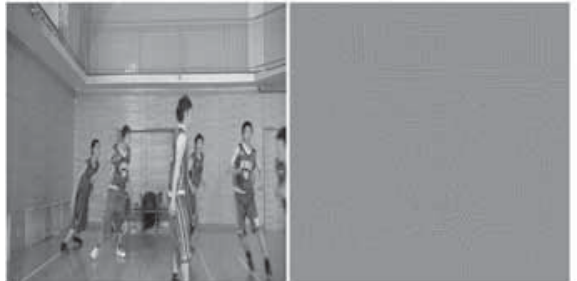

(a) Frame 306 of sequence BasketBall.yuv (left) and its RE residue (right)

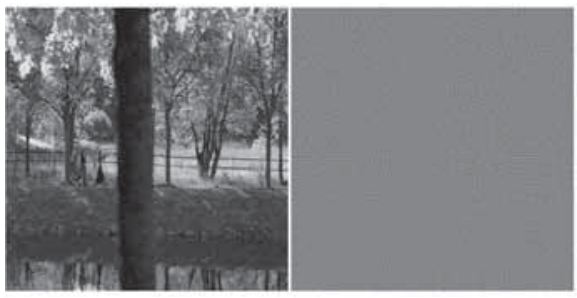

(c) Frame 6 of sequence ParkJoy.yuv (left) and its $\mathrm{RE}$ residue (right)

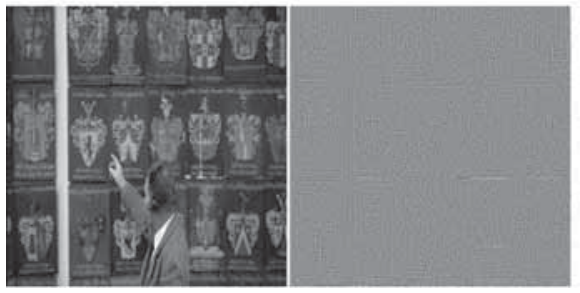

(e) Frame 6 of sequence Shield.yuv (left) and its $\mathrm{RE}$ residue (right)

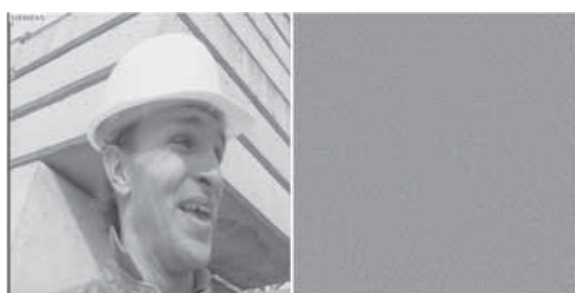

(b) Frame 6 of sequence Foreman.yuv (left) and its $\mathrm{RE}$ residue (right)

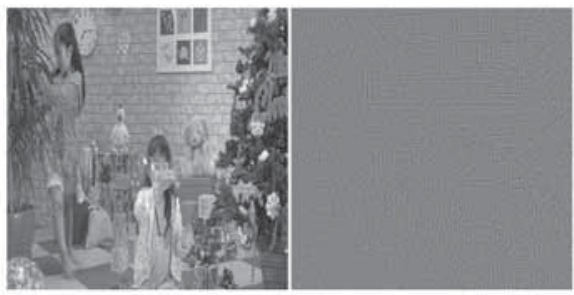

(d) Frame 6 of sequence Party.yuv (left) and its $\mathrm{RE}$ residue (right)

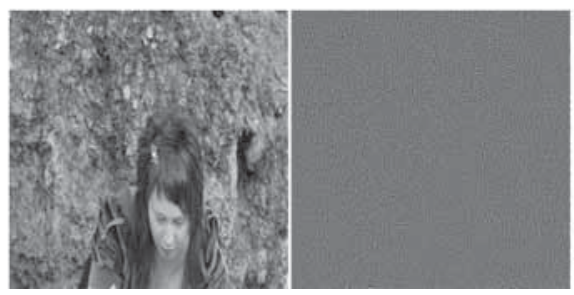

(f) Frame 306 of sequence TreeTilt.yuv (left) and its RE residue (right)

Figure 7. Resolution enhancement residuals for different test frames. The RE residuals are obtained from the spatially scalable extension of Dirac video codec as shown in figure 3.

\section{Results on residual coding using HWD-H}

Eslami's set of HWD transforms were reported to give promising results on images with more textures and detail information (Eslami \& Radha 2006). However, their use on residual frames was not investigated. HWD-H, although being non-redundant and non-adaptive, is capable of extracting features in multiple directions, a desired quality in coding of the residuals. This transform suits for our purpose of coding the RE residuals well. An example which demonstrates the application of the HWD transform on the Spokes image is depicted in figure 6. The transform being non-redundant, subbands after filtering are arranged in a specific fashion to ease the further process of encoding the coefficients. The details of the arrangement are given in the work of Eslami \& Radha (2007).

The comparison of HWD-H and DWT to encode RE residuals is carried out under equivalent conditions using the SPIHT algorithm (Said \& Pearlman 1996). It makes use of the parent-child 


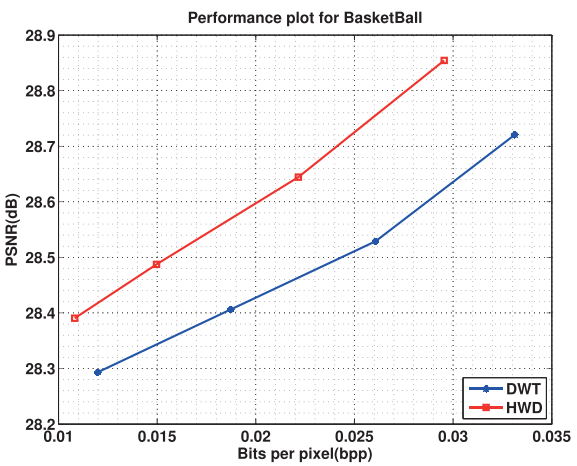

(a) Performance for frame 306 of sequence BasketBall.yuv

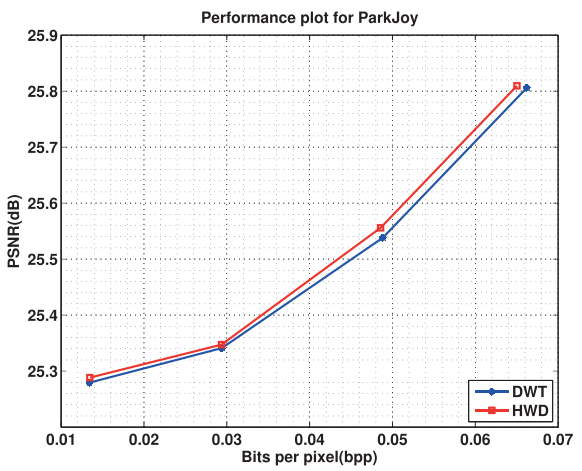

(c) Performance for frame 6 of sequence ParkJoy.yuv

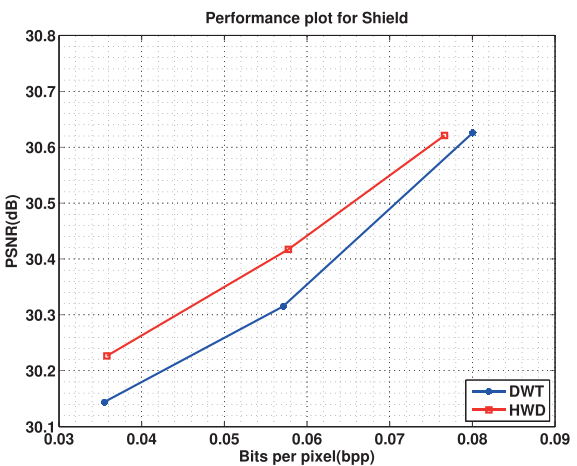

(e) Performance for frame 6 of sequence Shield.yuv

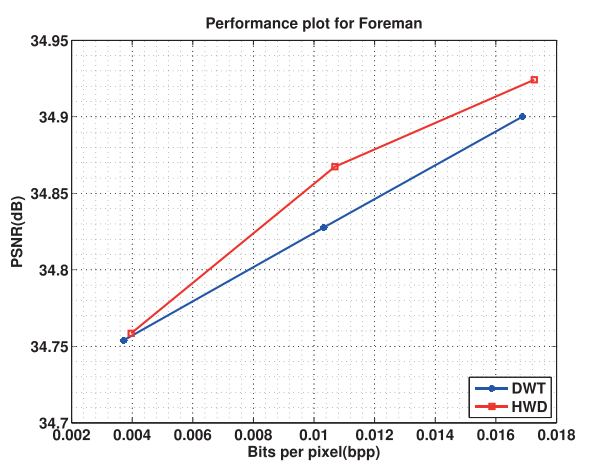

(b) Performance for frame 6 of sequence Foreman.yuv

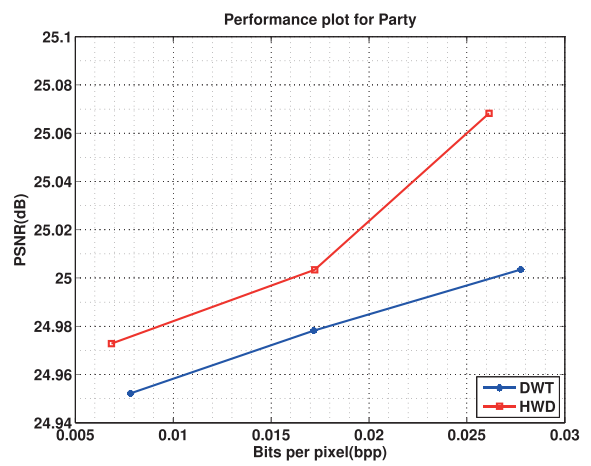

(d) Performance for frame 6 of sequence Party.yuv

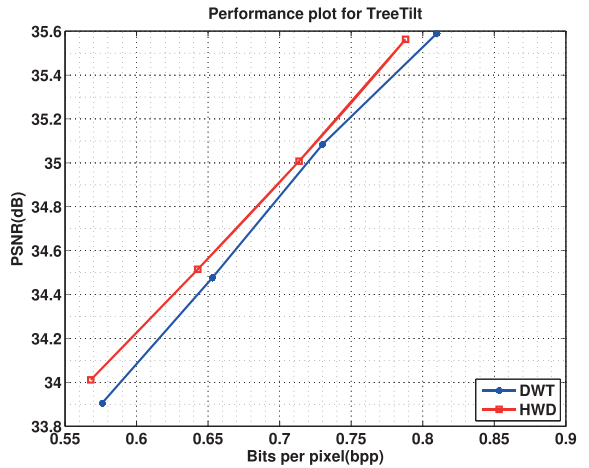

(f) Performance for frame 306 of sequence TreeTilt.yuv

Figure 8. PSNR performance of residual coding with a comparison of the suggested HWD-H with the traditional DWT bior4.4 filters. 
Table 1. Performance of HWD over DWT using PSNR $(\mathrm{dB})$ as metric.

\begin{tabular}{ll}
\hline Sequence & $\begin{array}{l}\text { PSNR } \\
\text { improvement }(\mathrm{dB})\end{array}$ \\
\hline BasketBall & 0.11 \\
Shield & 0.08 \\
TreeTilt & 0.06 \\
Foreman & 0.04 \\
Party & 0.03 \\
ParkJoy & 0.01 \\
\hline
\end{tabular}

self-similarity concept in wavelet decomposition. Frames extracted from six different test video sequences were tested. All the test frames chosen have dimensions $512 \times 512$. Residuals of luma components have significant directional information as compared to their croma counterparts. Therefore, for the purpose of evaluation, we have chosen only luma components of corresponding RE residual frames. The test frames (luma-only) and their corresponding RE residuals are shown in figure 7.

The decomposition vector used is $[2,2,0,0,0]$ which signifies the number of directional decompositions at each level. The orientation of frequency support for $L=2$ takes the values $\theta=$ $67.5^{\circ}, 112.5^{\circ}, 22.5^{\circ}$, and $-22.5^{\circ}$. The decomposition vector signifies initial five-level decomposition of normal DWT and two level directional decomposition on the subbands obtained in the first phase. No directional decomposition is applied on the subbands obtained at levels 3, 4, and 5. The effect of decomposition on frequency bands at different levels can be found in the reference (Li Ma et al 2010). The wavelet chosen for DWT coding and first stage of HWD$\mathrm{H}$ coding is the bior4.4. The PSNR performance of the reconstructed frame is shown in figure 8. An average PSNR improvement for HWD over DWT is shown in table 1. The results show better performance for residuals with higher directionality content like Basketball and Shield and Treetilt. Whereas for the sequence like Foreman, Party and Parkjoy, the performance improvement is comparatively low or marginal. This shows the preference to use HWD over DWT to encode residuals with higher directionality content, which is the approach suggested in this paper.

\section{Conclusion}

The RE residuals, generated from the inter-scale differences in a spatially scalable architecture, possess more directional properties as compared to the IP and MC residuals. We have used a class of directional and non-redundant transforms to encode the RE residuals. The simulations were carried out on a variety of test frames across the same platform of the SPIHT algorithm. The residuals were generated from Dirac video codec and sampling filters were adopted from H.264 JSVM. The results for RE residual coding show a slight improvement in the coding efficiency with HWD over DWT for the residuals with a high amount of texture, while there is hardly any improvement for other residuals. The performance of wavelet-based video codecs like Dirac can benefit from the use of directional transforms for residual coding to improve the efficiency of compression. 


\section{Acknowledgements}

The authors thank Mr. Ramin Eslami of Rochester University for providing the filter toolbox, Mr. Tim Borer of BBC, Prof. Fatih Kamisli of Middle East Technical University and Mr. Anant Malewar of Nex Robotics India, for their valuable inputs.

\section{References}

Chang S G, Cvetkovic Z and Vetterli M 1995 Resolution enhancement of images using wavelet transform extrema extrapolation. In: Acoustics, Speech, and Signal Processing, 1995. ICASSP-95., 1995 Int. Conf. 4: $2379-2382$

Chen C-F and Pang K K 1993 The optimal transform of motion-compensated frame difference images in a hybrid coder. Circuits and Systems II: Analog and Digital Signal Processing, IEEE Transactions 40(6): 393-397

Cohen RA, Klomp S, Vetro A and Huifang Sun 2010 Direction-adaptive transforms for coding prediction residuals. In: Image Processing (ICIP), 2010 17th IEEE Int. Conference 185-188

Eslami R and Radha H 2006 Regular hybrid wavelets and directional filter banks: Extensions and applications. In: Image Processing, 2006 IEEE Int. Conference 1609-1612

Eslami R and Radha H 2007 A new family of nonredundant transforms using hybrid wavelets and directional filter banks. Image Processing, IEEE Transactions 16(4): 1152-1167

Je-Won Kang, Gabbouj M and Kuo C-C J 2013 Sparse/dct (s/dct) two-layered representation of prediction residuals for video coding. Image Processing, IEEE Transactions 22(7): 2711-2722

Jianping Zhou, Cunha A L and Do M N 2005 Nonsubsampled contourlet transform: construction and application in enhancement. In: Image Processing, 2005. ICIP 2005. IEEE Int. Conference: I-469-72

Kalra V, Wahid K and Dinh A 2011 Video codec comparative analysis between h.264 and dirac pro/vc-2. In: Electrical and Computer Engineering (CCECE), 2011 24th Canadian Conference on, pages 000951000955

Kamisli F 2010 Transforms for Prediction Residuals in Video Coding. PhD thesis, Dept. of Electrical Engineering and Computer Science, M.I.T., Massachusetts

Kamisli F and Lim J S 2009a Directional wavelet transforms for prediction residuals in video coding. In: Image Processing (ICIP), 2009 16th IEEE Int. Conference 613-616

Kamisli F and Lim J S 2009b Transforms for the motion compensation residual. In: Acoustics, Speech and Signal Processing, 2009. ICASSP 2009. IEEE Int. Conference 789-792

Li Ma, Chunling Yang and Duanwu Cao 2010 32still image compression algorithm based on directional filter banks. Int. J. Information Technol. Comput. Sci. 2(2): 25-32

Niehsen W and Brunig M 1999 Covariance analysis of motion-compensated frame differences. Circuits and Systems for Video Technology, IEEE Transactions 9(4): 536-539

Rao K R, Britanak V and Yip P C 2007 Discrete cosine and sine transforms, general properties, fast algorithms and integer approximations, chapter 2, pages 51-72 Academic Press

Said A and Pearlman W A 1996 A new, fast, and efficient image codec based on set partitioning in hierarchical trees. Circuits and Systems for Video Technol. IEEE Transactions 6(3): 243-250

Schwarz H, Marpe D and Wiegand T 2007 Overview of the scalable video coding extension of the h.264/avc standard. Circuits and Systems for Video Technology, IEEE Transactions 17(9): 1103-1120

Segall C A and Sullivan G J 2007 Spatial scalability within the h.264/avc scalable video coding extension. Circuits and Systems for Video Technology, IEEE Transactions 17(9): 1121-1135

Selesnick I W, Baraniuk R G and Kingsbury N C 2005 The dual-tree complex wavelet transform. Signal Processing Magazine, IEEE 22(6): 123-151

Spokes image. [online]. http://www.silverwinggraphics.com/focustarget.htm. Accessed: 2013-07-29

Sullivan G J, Ohm J, Woo-Jin Han and Wiegand T 2012 Overview of the high efficiency video coding (hevc) standard. Circuits and Systems for Video Technology, IEEE Transactions 22(12): 1649-1668 
Temizel A and Vlachos T 2005 Wavelet domain image resolution enhancement using cycle-spinning. Electronics Lett. 41(3): 119-121

Ya-quin Zhang, Yao Wang and Joern Osterman 2001 Video Processing and Communications. Prentice Hall PTR, Upper Saddle River, NJ, USA 